\title{
Acute Rhino-orbital-cerebral Mucormycosis in a Patient with COVID - 19
}

\author{
Warda Ali Naqvi ${ }^{1}$, Muhammad Javid Bhutta ${ }^{2}$, Ejaz Ahmed Khan ${ }^{3}$, Aftab Akhtar ${ }^{4}$, Sania Raza ${ }^{5}$ \\ ${ }^{1-5}$ Shifa International Hospital, Islamabad
}

\begin{abstract}
COVID-19 patients are known to have immunosuppression due to decreased lymphocytes and increased susceptibility to co-infections (Bacterial and fungal). We present a case of 61 -year-old patient who had diabetes, hypertension and ischemic heart disease with COVID-19 infection admitted after RT-PCR positive result. He developed rhino-orbital Mucormycosis during treatment. He received Remdesivir with parenteral Methylprednisolone and Meropenem. While admitted in the ward, he developed signs of orbital cellulitis. Magnetic resonance imaging $(\mathrm{MRI})$ of the brain, orbits, and paranasal sinuses revealed right frontal, ethmoidal, and maxillary sinusitis with the extension of the sinisuidal disease to the orbit. A nasal biopsy revealed broad a septate filamentous fungal hyphae suggestive of Mucormycosis. Long-term use of steroids/monoclonal antibodies/broadspectrum antibiotics may contribute to the predisposition to fungal disease. Early diagnosis and prompt management are warranted to avoid morbidity.
\end{abstract}

Key Words: Mucormycosis, COVID - 19, Orbital Cellulitis.

How to Cite this Article: Naqvi WA, Bhutta MJ, Khan EA, Akhtar A, Raza S. Acute Rhino-orbital-cerebral Mucormycosis in a Patient with COVID - 19. Pak J Ophthalmol. 2022, 38 (1): 76-79.

Doi: $10.36351 /$ pjo.v38i1.1301

\section{INTRODUCTION}

Patients of COVID - 19 suffer immunosuppression due to decreased lymphocytes and hence they have an increased susceptibility to other infections. Published data shows that Diabetes mellitus, lung diseases, old age and critically ill patients requiring ventilator support are more prone to fungal and bacterial coinfections. ${ }^{1,2}$ Aspergillosis is widely suspected among fungal infections in COVID-19 patients. However, COVID - 19 associated Mucormycosis (CAM) are often under diagnosed and need to be understood with respect to the clinical features, outcomes, and risk factors for the early diagnosis of this lethal condition. ${ }^{3}$

Correspondence: Warda Ali Naqvi

Shifa International Hospital, Islamabad

Email:warda.ali91@yahoo.com

Received: June 15, 2021

Accepted: December 5, 2021
Mucormycosisisis a lethal, opportunistic, and invasive fungal infection seen in immunocompromised individuals. Around 30 to $50 \%$ of diabetic patients develop rhino-orbital-cerebral form (ROCM). However, the mortality due to extension to brain is about $100 \% .^{3}$ Tissue necrosis in Mucormycosis causes poor penetration of the antifungal therapy at the lesion site attributing to delayed treatment response. The prerequisites for diagnosing Mucormycosis are; a high index of suspicion and prompt assessment of clinical manifestations. ${ }^{2}$ So there is a need for early diagnosis of this highly deadly disease. ${ }^{4}$

Recently, there is a rise in fungal infections which is associated with COVID - 19. Pakistan shows a $15 \%$ increase in fungal complications of COVID $-19^{1}$ and warrants algorithms for early diagnosis and management of fungal infections, especially the lifethreatening ROCM. ${ }^{1}$ Here we present a patient with multiple comorbidities and COVID - 19, who developed acute rhino-orbital Mucormycosis, which 
disseminated to the brain and caused death of the patient.

\section{Case Presentation}

A 61-year-old male patient was admitted to the COVID - 19 critical ward with an eight-day history of shortness of breath, fever and dry cough. He had taken one dose of Tocilizumab before admission with seven days history of Azithromycin and Doxycycline use. He was a longstanding case of hypertension and diabetes (> 10 years), taking insulin (Aspart and glargine) and antihypertensive treatment (Carvedilol). He also had a history of percutaneous coronary intervention for the last two years and was using Amlodipine with aspirin. On examination, his pulse rate was $124 /$ minute, blood pressure was $130 / 78 \mathrm{mmHg}$, respiratory rate was $22 /$ minute with an oxygen saturation of $82 \%$ on room air which improved to $93 \%$ on 5 liters/minute with rebreather mask and he was a febrile on admission. The relevant physical examination revealed bilateral crepitation at the lung bases and no cardiovascular or neurological abnormalities.

A reverse-transcriptase polymerase chain reaction (RT-PCR) was positive for the SARS-CoV-2 virus. There were bilateral non-homogeneous opacifications on the chest X-ray and computed tomography (CT) scan of the chest showed multifocal, multi-lobar peripheral ground-glass opacities typical for COVID 19. Intravenous Methylprednisolone (40 mg twice daily), intravenous Meropenem (1g 8 hourly) and intravenous Remdesivir (200 mg for one day and $100 \mathrm{mg}$ per day as maintenance dose) were started. His pre-meal blood sugar was set at $180-200 \mathrm{mg} / \mathrm{dl}$ with insulin using sliding scale method. Subcutaneous Enoxaparin $(40 \mathrm{mg} / 0.4 \mathrm{ml})$ once daily and three doses of $6 \mathrm{mg}$ Ivermectin were also given as per the institution guidelines.

His oxygen demand gradually increased to 15 liters/min on rebreather mask over the next few days, upon which BiPAP was started with an increase in intravenous Methylprednisolone (60 mg twice daily). On day seven, the patient improved and was shifted to a regular COVID - 19 ward with oxygen 15 liters/min on the non-rebreather mask. Rest of the treatment was continued as before.

On day 9, the patient experienced severe headache associated with right eyelid swelling, proptosis and pain radiating to the forehead. He was advised topical Moxifloxacin and radiological tests by an ophthalmologist. The next day, an MRI and CT scan of the brain, orbits, and paranasal sinuses revealed right frontal, ethmoidal and maxillary sinusitis with mild thinning of the right-sided lamina papyracea of the ethmoid sinus. The disease had also extended to the orbit. After the Infectious Diseases consult, empiric treatment was initiated with liposomal amphotericin B (300 - mg/day). Isavuconazole (200 $\mathrm{mg}$ twice a day for 48 hours followed by $200 \mathrm{mg}$ once daily) was also added later on.

After two days, the right eye showed proptosis with periorbital edema and conjunctival congestion. The left eye appeared normal. Gross visual acuity was intact and ocular movements showed mild restriction in extremes of all gazes on the right side. CT scan was repeated which revealed the disease extent to the orbital apex with possible thrombosed right ophthalmic artery and small orbital abscess along the superomedial wall. ENT team did an urgent functional endoscopic sinus surgery (FESS) and orbital decompression on Day $13^{\text {th }}$. Histology and fungal cultures revealed broad a septate hyphae branching at 90 degrees, suggestive of mucor species confirmed on a Sabourauds Dextrose Agar culture.

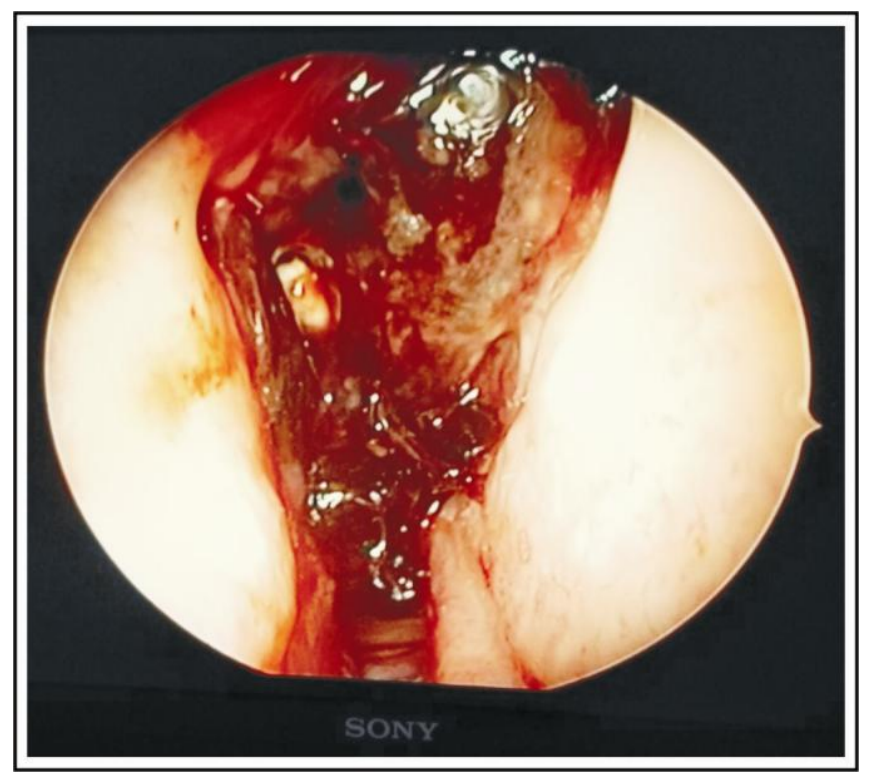

Fig. 1A: Intraoperative Aspect: Extensive necrosis and hemorrhage in the right ethmoid sinus.

Tissue cultures also revealed growth of Escherichia coli. Intravenous Meropenem was increased to $2 \mathrm{~g}$ three times a day. MRI brain and orbits with contrast after four days showed worsening of the 


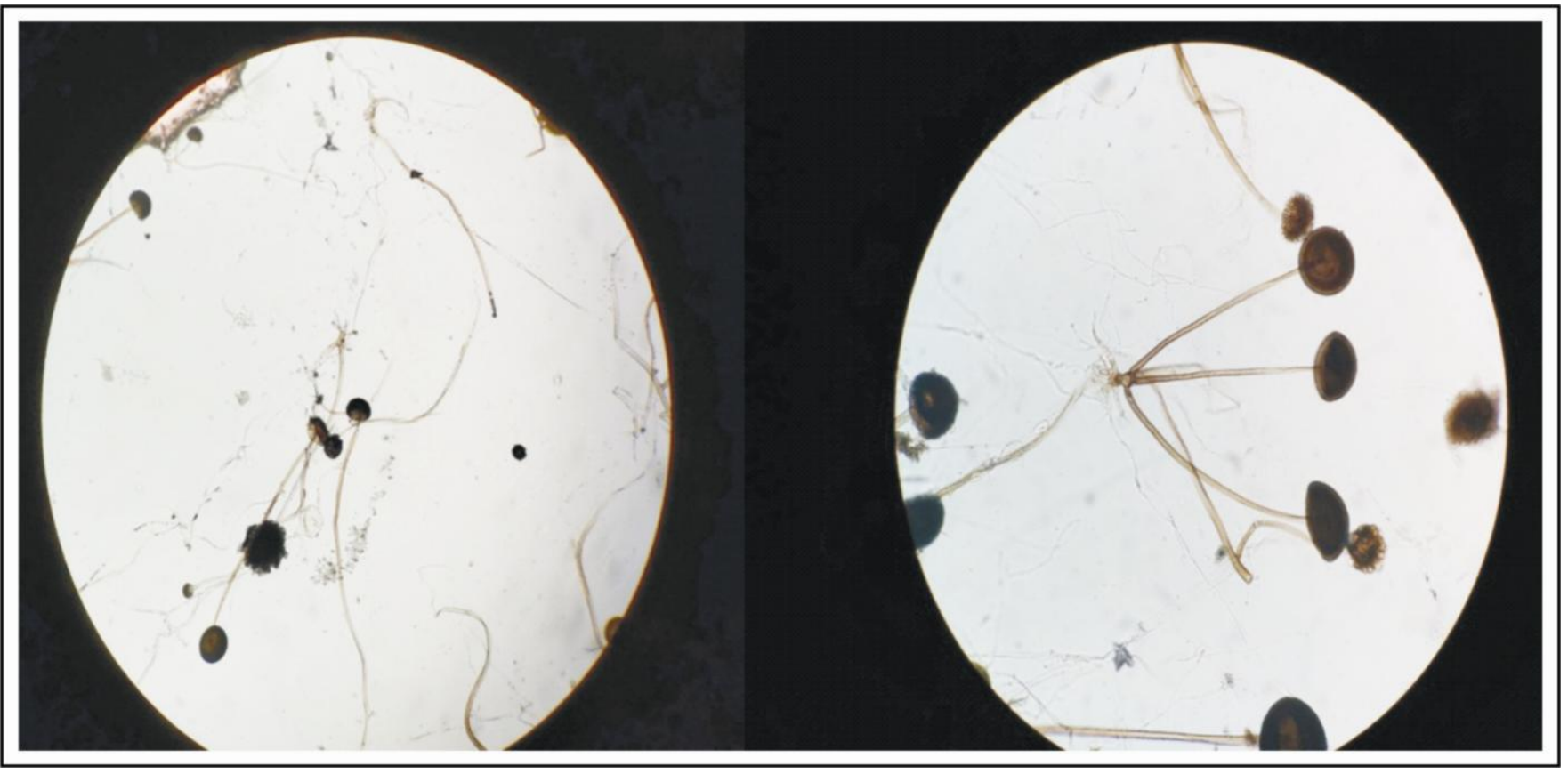

Figure 1B and 1C: A septate hyphae branching at 90 degrees, suggestive of Rhizopusspecies.

lesion with intracranial extension and asymmetric enhancement of dura on the right side, while intraorbital contents on the left side did not show any pathology. Meningio-encephalitis and involvement of the right cavernous sinus and the ophthalmic veins were also noted.

The patient continued to deteriorate, developed hypertensive emergency and was intubated for ventilator support. Later he required inotropic support over the next couple of days. He also developed renal failure. Due to multi-organ failure and the spread of the disease to the brain the patient expired on day thirty-six of admission.

\section{DISCUSSION}

The purpose of presenting this case is to highlight the challenging possibility of opportunistic fungal infections in COVID-19 cases and its management. Our patient not only had diabetes to contribute towards acquiring ROCM but also had hypertension, COVID19 and IHD. Rhino-orbital-cerebral mucormycosis is the most common disease form affecting up to $55 \%$ of the diabetic patients with COVID-19. ${ }^{2}$ There has been a marked increase in Mucormycosis in diabetic patients who develop COVID-19.,6

Werthman-Ehrenreich $\mathrm{A}^{4}$ reported a case of a 33year-old COVID-19 positive female with altered mental status and proptosis who had developed orbital Mucormycosis. It was suggested to highlight the early identification of this high morbidity disease for better outcomes. Other factors predisposing to Mucormycosis in COVID positive patients include prolonged steroid treatment and immunomodulatory drugs. Mehta $\mathrm{S}$ et al. shared a case of a 66-year-old male who had COVID-19 and developed rhino-orbital mucormycosis after steroids therapy. ${ }^{1}$ In COVID-19, the reduced numbers of $\mathrm{T}$ lymphocytes, CD4+T, and $\mathrm{CD} 8+\mathrm{T}$ cells, may alter innate immunity, causing predisposition to fungal infections along with the effects of the drugs.

Another case was reported by Waizel-Haiat S et al, in which a young patient with COVID, developed rhino-orbital Mucormycosis. ${ }^{7}$ They proposed that in diabetic patients, symptoms such as cranial nerve palsy, mid-facial pain, diplopia, proptosis, apex orbital syndrome, periorbital edema and palatine ulcer raise the red flags for ROCM. In another case report, the authors discussed the importance of early diagnosis and treatment of fungal co-infections in COVID-19. The prompt recognition of this complication and initiation of anti-fungal therapy are essential to decrease morbidity owing to fungal coinfections and extension into the adjoining structures. ${ }^{8}$

Other authors have also described the importance of early diagnosis, start of antifungal treatment and 
surgical debridement to prevent morbidity and mortality. ${ }^{9,10}$ Early administration of liposomal amphotericin through a retrobulbar injection with accompanied systemic antifungals in the setting of rhino-orbital-mucormycosis can also help patient survival.

\section{CONCLUSION}

Our report highlights the importance of early diagnosis of challenging possibility of ROCM which can help in starting prompt therapy resulting in better patient outcomes. Early diagnosis of ROCM through warning signs, ophthalmological symptoms can help initiation of early treatment.

\section{Conflict of Interest}

Authors declared no conflict of interest.

\section{REFERENCES}

1. Mehta S, Pandey A. Rhino-Orbital Mucormycosis Associated With COVID-19. Cureus, September 30, 2020; 12 (9): e10726. doi:10.7759/cureus.10726

2. Ashraf DC, Idowu OO, Hirabayashi KE, KalinHajdu E, Grob SR, Winn BJ, et al. Outcomes of a Modified Treatment Ladder Algorithm using Retrobulbar Amphotericin B for Invasive Fungal Rhino-Orbital Sinusitis. Am J Ophthalmol. 2021 Jun. 8: S0002-9394 (21): 00319-6.

Doi: 10.1016/j.ajo.2021.05.025. Epub ahead of print. PMID: 34116011.

3. Bartoletti M, Pascale $\mathbf{R}$, Cricca $M$, Rinaldi $M$, Maccaro A, Bussini L, et al. PREDICO Study Group. Epidemiology of Invasive Pulmonary Aspergillosis among Intubated Patients with COVID-19: A Prospective Study. Clin Infect Dis. 2021 Dec. 6; 73 (11): e3606-e3614. Doi: 10.1093/cid/ciaa1065. PMID: 32719848 ; PMCID: PMC7454393.

4. Werthman-Ehrenreich A. Mucormycosis with orbital compartment syndrome in a patient with COVID-19. Am J Emerg Med. 2021 Apr; 42: 264.e5-264.e8.

Doi: 10.1016/j.ajem.2020.09.032. Epub 2020 Sep 16. PMID: 32972795; PMCID: PMC7493738.

5. Fouad YA, Abdelaziz TT, Askoura A, Saleh MI, Mahmoud MS, Ashour DM, et al. Spike in RhinoOrbital-Cerebral Mucormycosis Cases Presenting to a Tertiary Care Center During the COVID-19 Pandemic. Front Med (Lausanne). 2021 May 28; 8: 645270. https://doi.org/10.3389/fmed.2021.645270

6. John TM, Jacob CN, Kontoyiannis DP. When Uncontrolled Diabetes Mellitus and Severe COVID-19 Converge: The Perfect Storm for Mucormycosis. J Fungi (Basel). 2021 Apr. 15; 7 (4): 298.

Doi: 10.3390/jof7040298. PMID: 33920755; PMCID: PMC8071133.
7. Waizel-Haiat S, Guerrero-Paz JA, Sanchez-Hurtado L, Calleja-Alarcon S, Romero-Gutierrez L. A Case of Fatal Rhino-Orbital Mucormycosis Associated With New Onset Diabetic Ketoacidosis and COVID-19. Cureus. 2021 Feb; 13 (2): e13163. doi:10.7759/cureus.13163

8. Maini A, Tomar G, Khanna D, Kini Y, Mehta H, Bhagyasree V. Sino-orbital mucormycosis in a COVID-19 patient: A case report. Int J Surg Case Rep. 2021 May; 82: 105957. Doi: 10.1016/j.ijscr.2021.105957

9. Ahmadikia K, Hashemi SJ, Khodavaisy S, Getso MI, Alijani N, Badali H, et al. The double-edged sword of systemic corticosteroid therapy in viral pneumonia: A case report and comparative review of influenza-associated mucormycosis versus COVID-19 associated mucormycosis. Mycoses, 2021 Aug; 64 (8): 798-808. Doi: 10.1111/myc.13256. Epub 2021 Mar 5. PMID: 33590551; PMCID: PMC8013756.

10. Emilin Pandian MD. The Role of Retrobulbar Liposomal Amphotericin in Orbital Apex Syndrome in a COVID-19 Positive Diabetic. J Med Clin Case Repo. 2021; f650: 804-9270.

\section{Author's Designation and Contribution}

Warda Ali Naqvi; Medical Officer: Concepts, Design, Literature Search, Data Acquisition, Manuscript Preparation, Manuscript Editing, Manuscript Review.

Muhammad Javid Bhutta; Consultant Infectious Diseases: Concepts, Design, Literature Search, Data Acquisition, Manuscript Preparation, Manuscript Editing, Manuscript Review.

Ejaz Ahmed Khan; Consultant Infectious Diseases: Concepts, Design, Literature Search, Data Acquisition, Manuscript Preparation, Manuscript Editing, Manuscript Review.

Aftab Akhtar; Head of Department: Concepts, Design, Literature Search, Data Acquisition, Manuscript Preparation, Manuscript Editing, Manuscript Review.

Sania Raza; Associate Consultant: Concepts, Design, Literature Search, Data Acquisition, Manuscript Preparation, Manuscript Editing, Manuscript Review. 